\title{
A Unified Explanation of Quantum Phenomena? \\ The Case for the Peer-to-Peer Simulation Hypothesis as an Interdisciplinary Research Program Marcus Arvan \\ University of Tampa
}

Abstract: In my 2013 article, "A New Theory of Free Will”, I argued that several serious hypotheses in philosophy and modern physics jointly entail that our reality is structurally identical to a peer-to-peer (P2P) networked computer simulation. The present paper outlines how quantum phenomena emerge naturally from the computational structure of a P2P simulation. §1 explains the P2P Hypothesis. §2 then sketches how the structure of any P2P simulation realizes quantum superposition and wave-function collapse (\$2.1.), quantum indeterminacy (§2.2.), wave-particle duality (§2.3.), and quantum entanglement (§2.4.). Finally, $\S 3$ argues that although this is by no means a philosophical proof that our reality is a P2P simulation, it provides ample reasons to investigate the hypothesis further using the methods of computer science, physics, philosophy, and mathematics.

Quantum mechanics is an exquisitely well-verified theory of how our physical world operates at a microscopic level. However, it presents us with two very deep puzzles: one of interpretation and one of explanation. First, there is the question of how to interpret its equations. Although there are dozens of different interpretations of the equations of quantum mechanics, every mainstream interpretation appears problematic. ${ }^{1}$ Second, there is the much deeper-and less well-understood-question of not just interpreting its equations, but of explaining why the world is quantum mechanical, that is, why the world operates according to such bizarre laws of nature. For, make no mistake about it, quantum mechanics is bizarre. According to quantum mechanics, microscopic objects such as electrons:

A. Appear ${ }^{2}$ to have properties of particles and of waves (wave-particle duality),

B. Can affect one another instantaneously across arbitrarily large distances (quantum entanglement),

\footnotetext{
${ }^{1}$ See e.g. Schlosshauer (2013) and Arvan (2013): §3.

2 Important note: not all interpretations of the formalism of quantum-mechanics imply that microscopic entities in fact have such properties. Some (e.g. Bohr) have suggested that we should merely think of quantum entities as either particles (e.g. in energy exchange) or waves. My paper does not assume a naïve realist view that quantum particles in fact have such properties. It is consistent both with $(\mathrm{A})$ an anti-realist interpretation of observations and the formalism (e.g. we should think of quantum entities as though they have features of particles and waves), as well as (B) a realist interpretation (viz. quantum entities in fact have the properties of each). I thank an anonymous reviewer for pressing this point.
} 
C. Exist in many different states - different locations, different spins - all at once (quantum superposition ${ }^{3}$ ),

D. Come to occupy determinate states only when measured (wave-function collapse ${ }^{4}$ ), such that, finally,

E. Where a given object (e.g. electron) will be measured to be can, in principle, only be predicted probabilistically prior to measurement (quantum indeterminacy)

It is commonly recognized, both in physics and the philosophy of physics, that we have no idea why the world has these features.

The present paper (1) sketches a unified explanation of these phenomena using the new model of reality that I proposed and defended in, "A New Theory of Free Will"5, for the purpose of (2) motivating further empirical research on the hypothesis (via methods of computer science, physics, philosophy, and mathematics). Before proceeding, I wish to emphasize point (2). Developing and empirically testing this new model of reality-the P2P Simulation Hypothesis-will require immense resources: interdisciplinary work by physicists, computer scientists, mathematicians, and philosophers to construct and analyze the mathematical and causal structures of P2P simulations, to see how they might need to be programmed replicate the specific quantum features of our reality (e.g. the specific mathematics of the quantum wavefunction, etc.). The present paper merely aims to motivate the development and empirical testing of the P2P Hypothesis as a philosophical and empirical project. My aim is to (1) sketch

\footnotetext{
${ }^{3}$ Important note: only some interpretations of quantum formalism imply superposition (e.g. the Copenhagen and Everett/many-worlds interpretation) - though these are the dominant interpretations today (see Schlosshauer et al. 2013). Some other interpretations (e.g. Bohmian Mechanics) interpret the same features of quantum mechanics in terms of "hidden variables", with no true superposition of states (see e.g. Cushing's 1994 discussion of Bohmian Mechanics). I will assume superposition for the sake of this paper, and thank an anonymous reviewer for pressing this point.

${ }^{4}$ It is also the case that only some interpretations (e.g. the Copenhagen interpretation) posit the wavefunction collapse, whereas other interpretations (e.g. Bohmian Mechanics) do not. I assume wave-function collapse for the sake of this paper, and thank an anonymous reviewer for pressing the point.

${ }^{5}$ Arvan (2013).
} 
how the P2P Hypothesis appears capable of providing the first unified explanation of quantum phenomena, thereby (2) motivating further philosophical and empirical investigation.

$\S 1$ of this paper briefly lays out the P2P Hypothesis, taking my first defense of it in "A New Theory of Free Will" as a starting point. §2 then sketches how the P2P Hypothesis promises to provide the first unified explanation of quantum superposition and wave-function collapse (§2.1.), quantum indeterminacy (§2.2.), wave-particle duality (§2.3.), and quantum entanglement (§2.4.). Finally, §3 argues that although this sketch is by no means a proof of the P2P Hypothesis, it provides ample reason to investigate the hypothesis more deeply using the methods of computer science, physics, mathematics, and philosophy.

\section{§1. The P2P Hypothesis}

Online computer simulations are by now familiar parts of our world. Computer scientists and videogame companies have created sophisticated simulated environments in which "players" can navigate and interact with one another online. These simulated environments often have, within them, functional analogues of the kinds of ordinary objects we interact with in our world: they have simulated rocks, simulated cars, simulated guns, simulated bullets etc. There are, however, two distinct types of online simulations: (1) "dedicated-server" simulations, and (2) peer-to-peer (P2P) simulations. Allow me to explain the difference. A dedicated server online simulation is one in which one computer on the network (the "dedicated server") represents where objects are in the simulated environment (see Figure 1). Every object in a dedicated server simulation thus has determinate properties within the simulation, including determinate positions and velocities. Moreover, provided the other computers hooked up to the simulation interact with the dedicated server properly, each computer on the network will take the same measurements, measuring objects in the simulated environment as having precisely the properties (e.g. location, velocity, etc.) represented on the server. 
Figure 1. A Dedicated Server Simulation

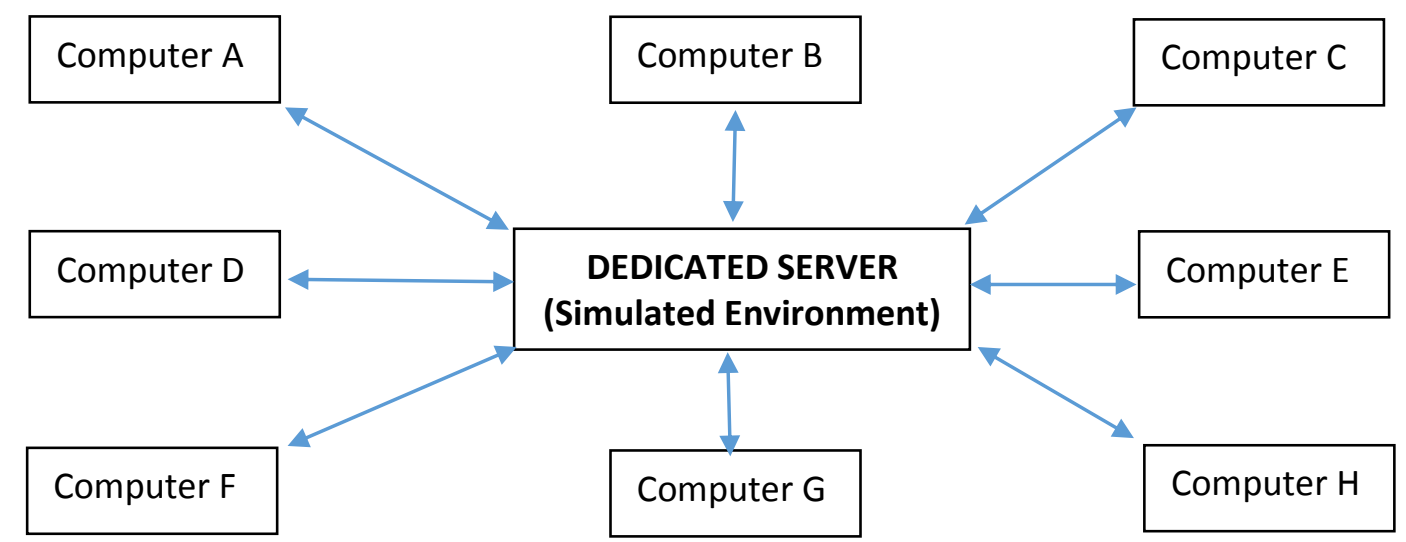

A peer-to-peer (P2P) networked simulation, however, is very different. In a P2P network, no single computer anywhere on the network encodes where objects in the simulated environment "objectively" are. Rather, the simulated environment is comprised by the entire network of computers on the network, each of which takes independent measurements at every instant, measurements which, in turn, at every successive instant, alter the measurements that other computers on the network will make (see Figure 2). In other words, a P2P simulation simply is an array of computers networked together where (A) each computer simulates the environment in parallel to every other computer on the network, and (B) the totality of individual measurements of each machine on the network at any given instant represents "the simulated environment" in which all computers on the network “experience in common." 
Figure 2. A Peer-to-Peer Network Simulation

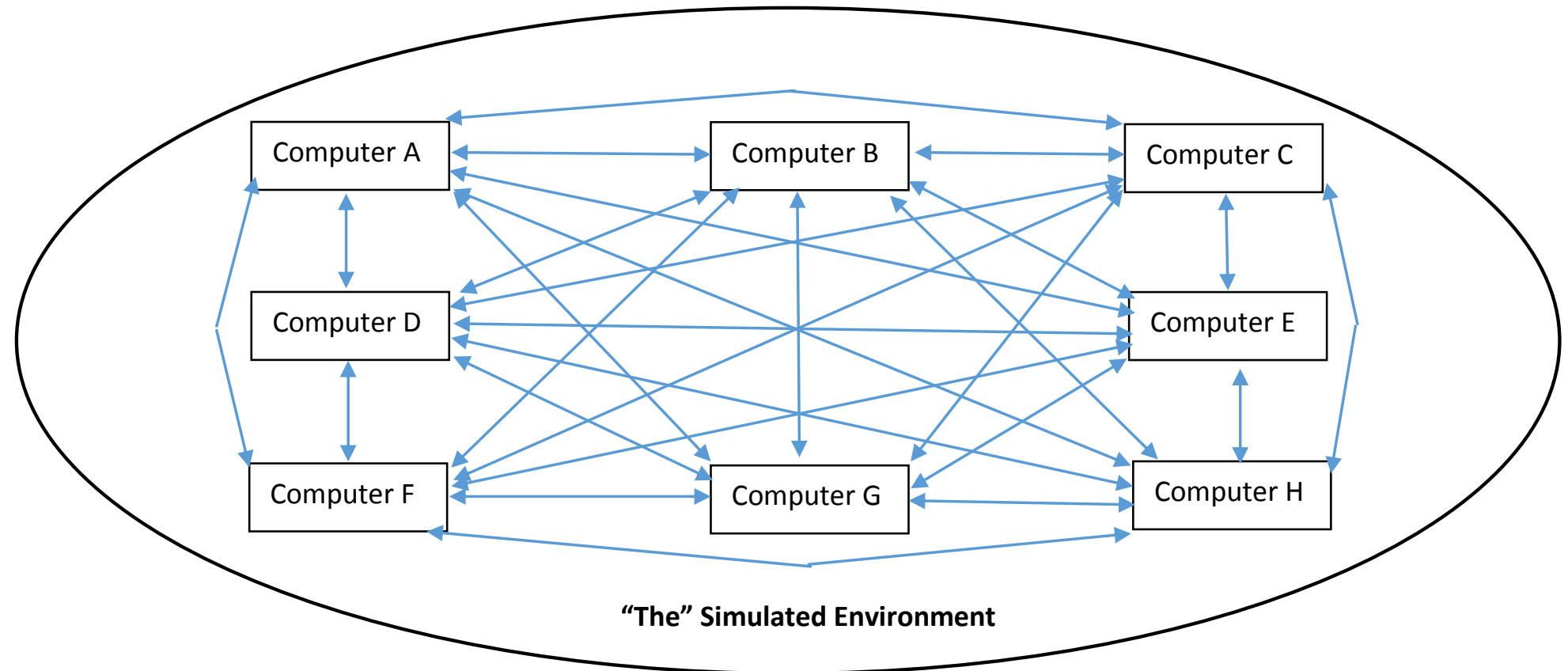

Now, very few online videogames in existence today actually utilize a P2P structure. The reason for this has to do with processing power and associated difficulties in achieving consistent error-correction. Here is the basic difficulty. In a P2P network, because each individual computer on the network represents "reality" in parallel, without effective error correction inconsistencies and violations of "causality" may occur across different machines. So, for example, if users on Computer A and B (above) are engaged in a simulated gunfight within "the" simulated environment, without proper error correction it can turn out that (1) computer A represents its user as “shooting and killing” computer B's user before the user of B can even get a shot off, but (2) computer B represents its user as "shooting and killing" computer A's user before the user of A can get a shot off. In other words, different computers on the network may represent mutually inconsistent versions of "reality." Second, these inconsistent representations may also appear t violate causality. If computer B represents its user as "shooting and killing" computer A's user but, due to some corruption in information 
transfer, computer A gets the "A is killed" information from B before it gets "A is shot" from B, computer A may represent its user as dying before even getting shot by B - an apparent violation of causality (i.e. an effect occurring before its cause).

It is important to realize that all of these problems are surmountable-that is, that workable $\mathrm{P} 2 \mathrm{P}$ simulations are possible-in principle, provided the computing system in question has enough processing power and meticulous error-correction. This is crucial, because although we may lack the computing power to make many effective P2P simulations (some online games today do utilize P2P networking effectively by requiring each player on the network to make a "move" in the game before the entire simulation updates), our universe, obviously, has an immense amount of computing power; enough, in principle, for our universe to be a P2P simulation. Moreover-and very interestingly-physicist James Gates has discovered clear analogues to error-correcting codes embedded within a string-theoretic analysis of the actual equations of quantum mechanics. ${ }^{6}$

Here, then, is what I would like to do. I want to assume-following my argument in "A New Theory of Free Will" - that it is epistemically possible that our universe is structurally identical to a P2P simulation: that, given our current knowledge, our world could be a P2P simulation (I defend this possibility in detail in my 2013 article; I will not review the arguments here). What I want to do then is explore what this hypothesis - the P2P Hypothesis - explains. As we will now see, it promises to explain all of the puzzling features of quantum mechanics mentioned earlier. The kinds of quantum phenomena we perceive inevitably arise within any peer-to-peer network.

${ }^{6}$ Gates (2010) 


\section{§2. How the P2P Hypothesis Promises a Unified Explanation of Quantum Phenomena}

Let us return to the strange quantum features of our world - features for which, again, we presently have no physical or metaphysical explanation.

\section{§2.1. The P2P Explanation of Quantum Superposition and Wave-function Collapse}

The essential features of quantum superposition are quite simple. The equations of quantum mechanics imply that objects in our world-for instance, electrons-simultaneously exist in many different states (e.g. locations) at once, prior to measurement: for instance, in locations A, B, C, D, and E. As strange (or even metaphysically impossible) as this phenomenon may sound at first glance, it is an absolutely fundamental principle of quantum mechanics, embodied in the mathematics of the Shrödinger equation. According to the Shrödinger equation, the electron really is in locations $\mathrm{A}, \mathrm{B}, \mathrm{C}, \mathrm{D}$, and $\mathrm{E}$ until it is measured, at which point the measurement itself will result in the electron coming to occupy one of the measured values (say, location B)-the "wave-function collapse" of the superposition. Although I have merely explained the phenomena of superposition and wave-function collapse here in simple terms, the explanation I have provided is mathematically accurate.

Let us now think again, however, about the structure of an ordinary P2P simulation. Recall that a P2P simulation is nothing more than many different computers-each of which has its own representation of reality-linked together exchanging information. Because of this-because each computer has its own representation of reality-unless the computers on the network have absolutely perfect error-correction algorithms, different computers on the network will inevitably represent objects in their environment as having slightly different properties, including locations. So, for instance, consider the physical location of a flying bullet in a P2P simulation at some given time. Computer A on the network may represent the bullet 
as being at location A, Computer B may represent it as being at a (slightly different) location B, and so on, across the entire array. In other words, we will have something like the following:

Figure 3. An Object's “Location” in a P2P Simulation

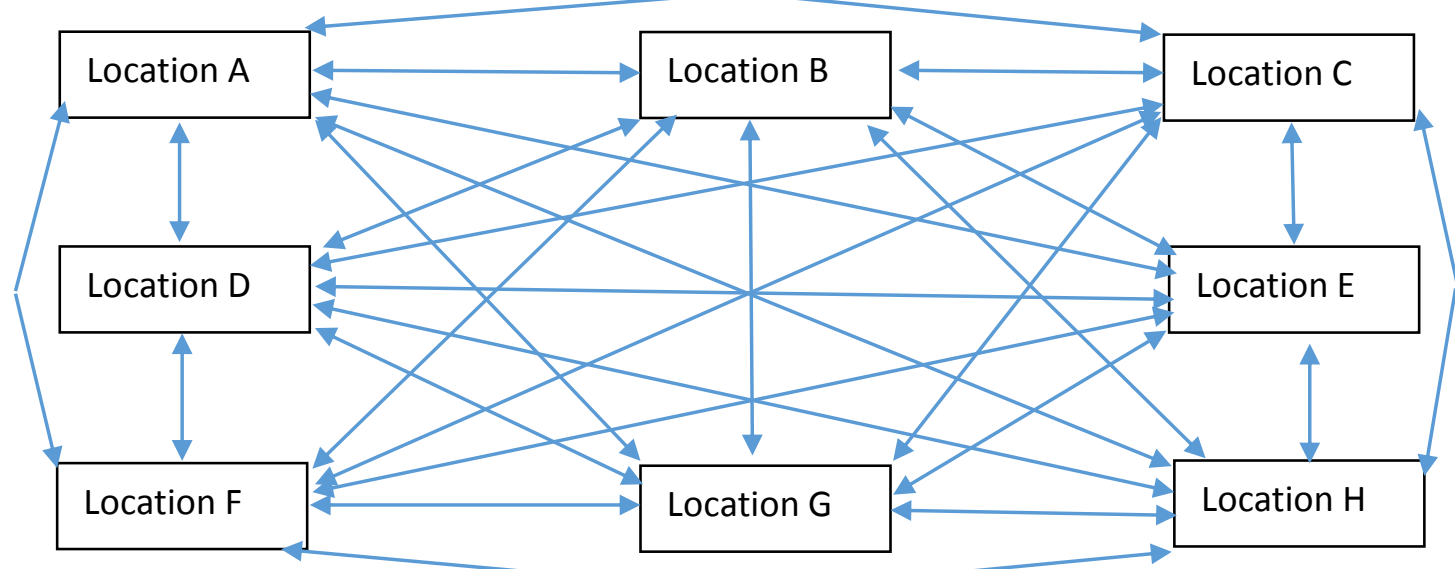

"The" Object's Objective Location in the Simulation = superposition of Locations A-H

The Object's Measured Location = A (by measurement apparatus A) B (by measurement apparatus B), etc.

Notice what is going on here. A P2P Simulation just is:

a. A superposition of different representational states, such that

b. Any particular measurement within the simulation will result in a determinate measured location on any individual computer taking a measurement.

But this is, functionally speaking, precisely what quantum superposition and wave-function collapse are in our world. Objects in our world exist in superposition, except that whenever they are measured, the measurement will result in a single determinate value. The P2P Hypothesis thus explains quantum superposition and wave-function collapse.

\section{§2.2. The P2P Explanation of Quantum Indeterminacy}

A second puzzling feature of quantum mechanics is that one cannot know, prior to taking a measurement, precisely where an object like an electron will be at the next instant. According 
to the laws of quantum mechanics-which, again, have been confirmed through observationelectrons literally "jump around" from point to point according to the quantum wave-function: one can only specify probabilistically what the chances are that a given electron will be measured to occupy one location rather than another (the wave-function specifies the relevant probabilities).

With this basic point in mind, let us turn to the dynamics of an ordinary P2P simulation. Thus far, the pictures I have drawn of the structure of P2P simulations (e.g. Figures 2 and 3) have been "snapshots" the structure of a P2P simulation at a specific point in time. As we have seen, at any particular point in time, a P2P simulation is a superposition of many different states, such that each particular measurement by a particular measuring device will result in the observation (relative to that device) of determinate properties of the object measured. So far, so good. What we have not yet discussed, however, is the dynamics of P2P simulations: that is, how different devices on a P2P network interact with and update one another.

Provided the P2P network in question has relatively good error-correction algorithms, measured values of objects across the simulation as a whole will tend to cluster probabilistically around central values. So, for instance, although one computer on the simulation may measure a bullet as existing at location $\mathrm{A}$ at time $\mathrm{t}$, a larger proportion of computers on the network may represent the bullet as existing at location B at that time. Then, there will also be some proportion of computers on the network that represent the bullet as being at location $\mathrm{C}$, some proportion at location $\mathrm{D}$, and so on - as follows: 
Figure 4. Sample Distribution of Measurements of an Object's Location in a P2P at time t

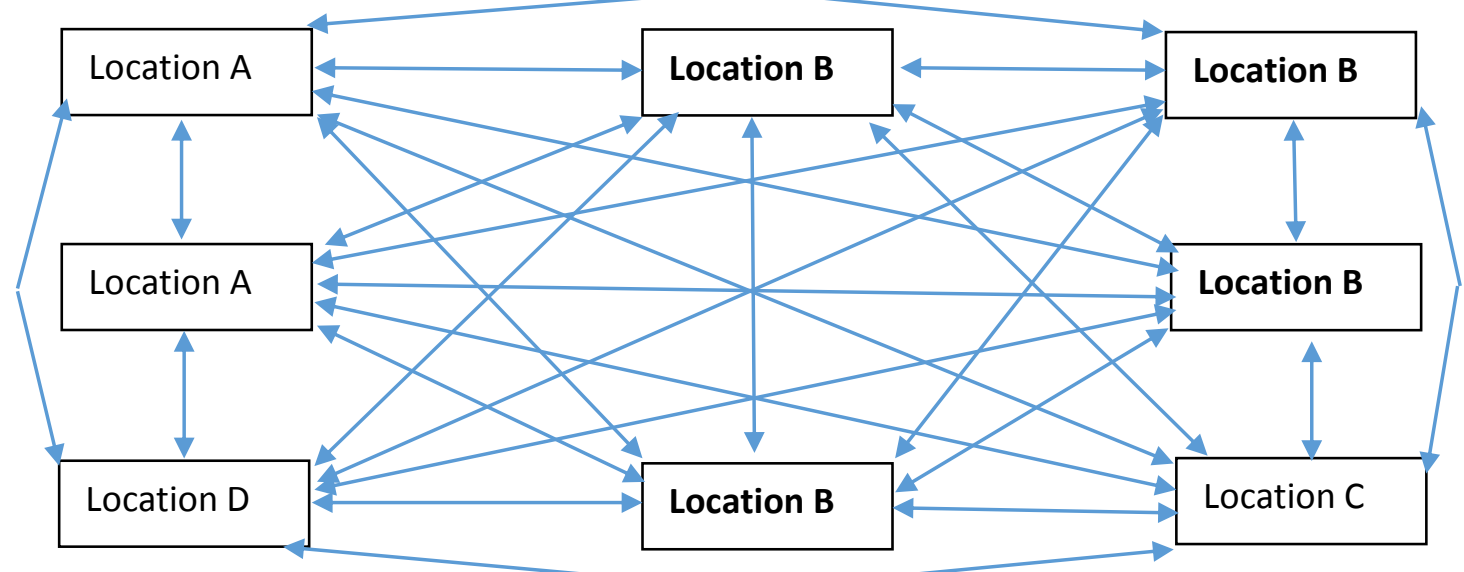

Here, next, is the crucial thing. At the next instant, each computer on the simulation trades its information with the others on the network. Each computer will "learn", for instance, that there are four computers on the network that represent the bullet as being as Location $B$, that there are two on the network that represent it as being at Location $\mathrm{A}$, and that there is one computer on the network that represents it as at location $\mathrm{C}$ and another sole computer representing it as being at located at position D. The task then for each computer on the network is to compute-on its own-is (A) where other computers on the network are going to represent the bullet's location at the next instant, and (B) plot that location correctly. But how is each computer on the network to do this? It must do it in light of the information it received from other computers on the network at time $t$, but also at earlier times (e.g.t-1). And here is the problem: every previous instant in the simulation will be another superposition of multiple representations, as in Figure 5: 
Figure 5. An Object's Location in a P2P Simulation at t-1

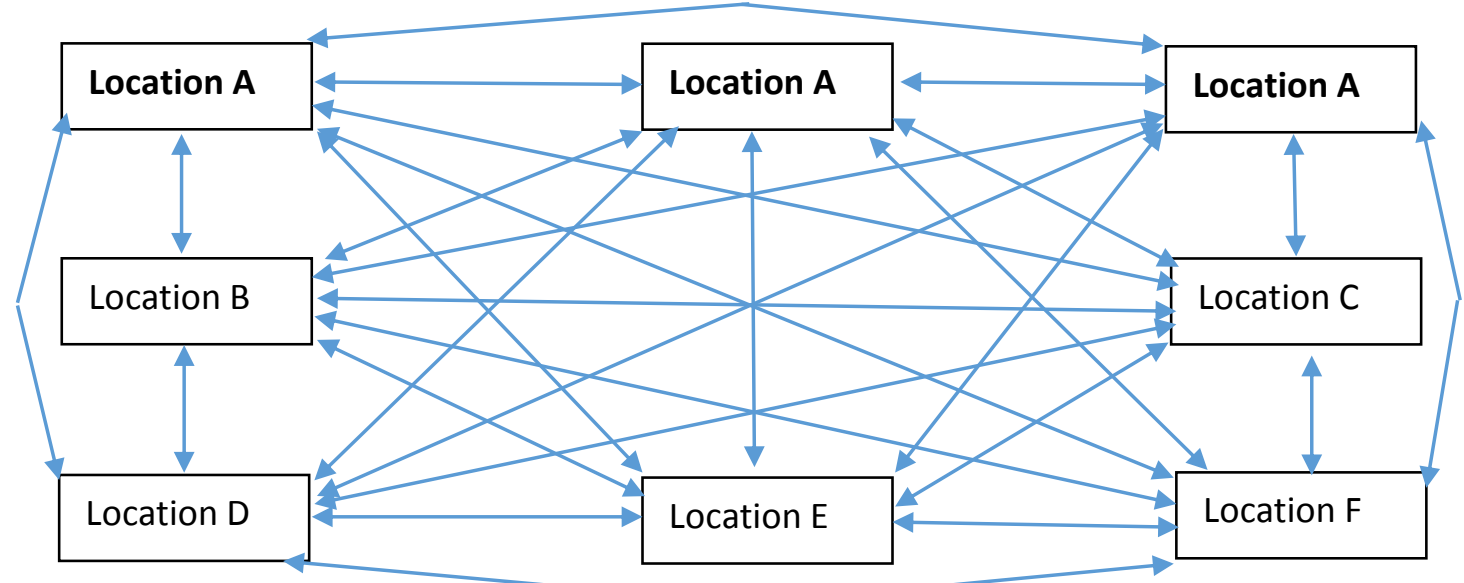

Notice, schematically, what is going on here. The "top three" computers or measurement devices all measure the bullet as being at location A at time $t-1$. Then, however, at time $t$ (Fig. 4), two of the computers in the top row represent the bullet at being at Location B, whereas the third represents it as still being at Location A. Furthermore, at $t$, two other computers on the simulation-one which represented the bullet as being in position $\mathrm{C}$ at $t-1$ and another which represented it as being at Location $\mathrm{E}$ at $t-1$-now agree with two computers in the top row about where the bullet is now located (Location B).

Here is the problem: from time $t-1$ to time $t$-at the level of the simulation as a wholethere is no single determinate "path" of the bullet through the simulation. Some computers which represent the bullet at Location B at $t$ now represented it earlier at Location $\mathrm{A}$, whereas others represented it as being at Locations C and E. Next, suppose the following is are computational laws within the simulation:

If object is in successive locations $\mathrm{A}$ then $\mathrm{B}$, plot object at location $\mathrm{X}$ at the instant.

If object is in successive locations $\mathrm{C}$ then $\mathrm{A}$, plot object at location $\mathrm{Y}$ at next instant.

If object is in successive locations $\mathrm{E}$ then $\mathrm{A}$, plot object at location $\mathrm{Z}$ at next instant. 
Provided the simulation has laws like this, no individual computer on network will be able to plot, deterministically, where the bullet will be at the next instant $(t+1)$. Rather, each computer will have to plot the bullet's location probabilistically (each computer can expect that there will be some proportion of computers on the network that represent the bullet at $\mathrm{X}$, some proportion that represent it at $Y$, etc.).

Thus, although at every instant a particular measurement on a P2P network can be expected to "collapse" the superposition of states to a single determinate measured value or location (the lesson of §2.1), dynamically, that is, over time, each computer on the network can only guess probabilistically where to properly plot the location of objects, the result of which is a new superposition of different states (e.g. at $t+1$ ). Accordingly, the P2P hypothesis explains quantum indeterminacy. An inability to predict deterministically where an object will be within a simulation-and a corresponding ability to only predict where an object will be probabilistically—is an inherent, emergent feature of peer-to-peer networking.

\section{§2.3. The P2P Explanation of Wave-Particle Duality}

Another puzzling feature of quantum mechanics is that, according to its equations, quantum phenomena (e.g. all particles) simultaneously have properties of particles and of waves. This seems paradoxical, of course, because to be a particle is to be localized (viz. existing at location A) whereas waves are spread out over space and time (viz. a wavelength is just that: something, that, in some sense, moves or propagates).

The P2P Hypothesis provides, however, a coherent, ready explanation of precisely how objects in any P2P networked environment can indeed have properties simultaneously. For, the dynamical progression of the following P2P system: 
Figure 6. Dynamical Progression in a P2P Network

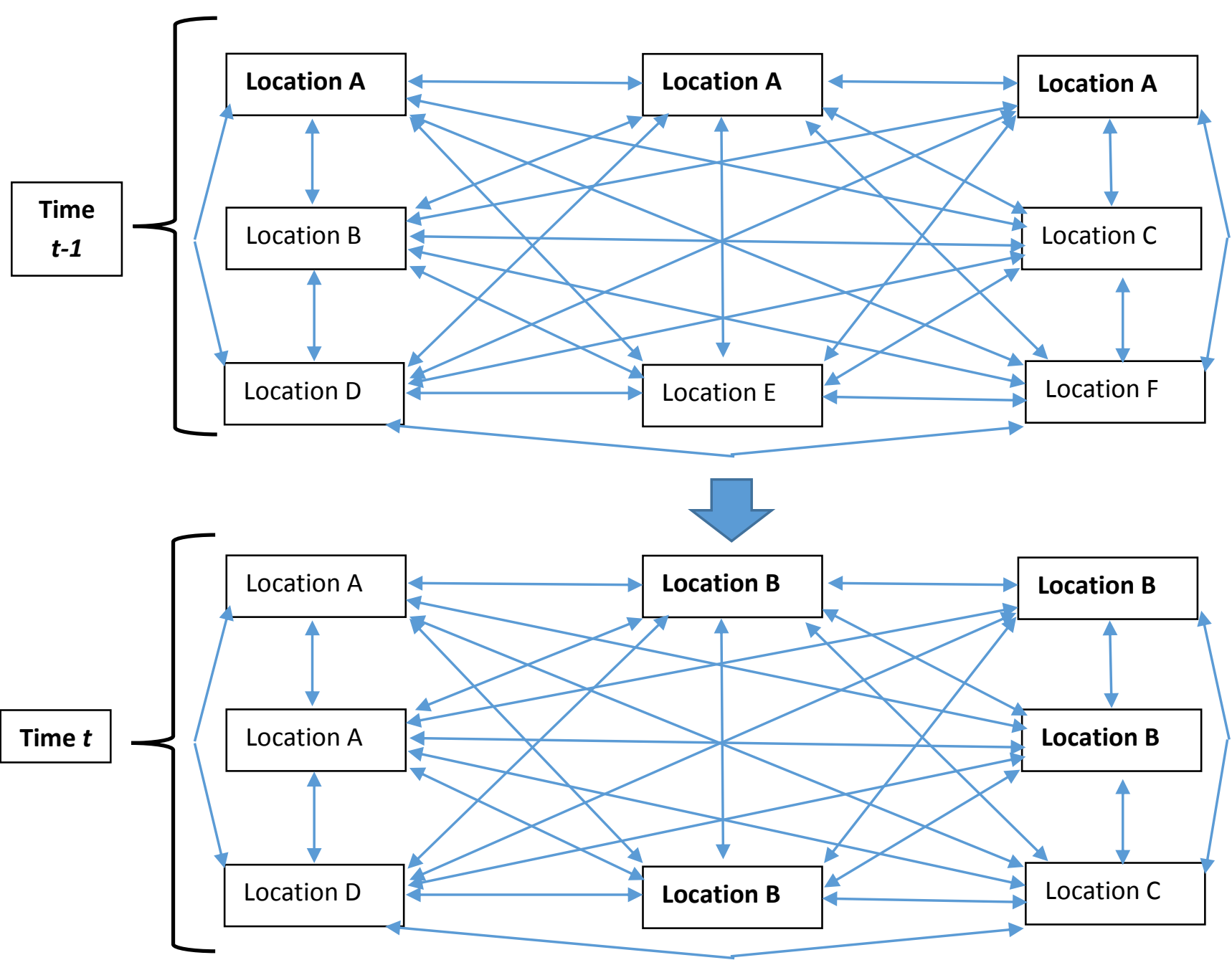

Notice how we can describe "the bullet" in this system. On the one hand, every individual computer processes it as a particle: as having a determinate location when measured by any individual computer. However, at the level of the system as a whole-and in terms of making predictions - the bullet's location also has the telltale properties of a wave. Because, at time t1, three computers on the network represent it as being in Location A, whereas only one computer represents it at being at Location B, a series of measurements at that time will result in an amplitude (i.e. three " $\mathrm{A}$ " measurements and only one "B" measurement). Second, the dynamical progression from $t-1$ to $t$ will result in measurements that comprise a wavelength (some "A" measurements at $t$-1 become $\mathrm{B}$ measurements at $t$, while one measurement remains 
at "A"). Thus, although (1) at every instant of measurement, each object in a P2P simulation has the properties of a particle (each measurement will result in an object occupying a determinate location), (2) the dynamics of the P2P simulation will cause any series of measurements to embody aspects of a wave (i.e. amplitude and wavelength/frequency). Thus, the P2P Hypothesis explains wave-particle duality.

\section{§2.4. The P2P Explanation of Quantum Entanglement}

Finally, we turn to the baffling feature of our world known as quantum entanglement. Schematically speaking, quantum entanglement is simple. Observations have shown that if we shoot photons at a "half-silvered" mirror in the right way, a single photon in effect becomes two entangled photons: one that passes through the mirror, and one that reflects back. ${ }^{7}$ The baffling thing about the "two" resulting photons is that, physically speaking, they are one: they are governed by precisely the same quantum wave-function, such that any quantum alteration to one of the entangled photons results in an instantaneous, corresponding alternation in the other photon, no matter how far apart the particles are, without any observable information transfer between them. In other words, quantum mechanics implies-and experiments have verified-that, essentially, one particle can simultaneously be in places at once.

We simply do not have any good explanation of why this is, or what mechanism could account for this (beyond the quantum wave-function itself)—that is, until now. For consider again the dynamics of a P2P simulation. For the sake of simplicity, let us simply take two computers joined together in a two-computer P2P network. Let us suppose that both computers represent, at time $t$, a single photon flying towards a half-silvered mirror. As we have already seen, these two computers on the network may represent that photon as having slightly different locations. Let us suppose, then, that prior to hitting the half-silvered mirror,

\footnotetext{
${ }^{7}$ See Brooks (2013) for a brief introduction.
} 
computer A represents the photon as being at Location A and Computer B represents the photon as being at Location $\mathrm{B}$, as in the following diagram:

Figure 7. Two P2P-Linked Computers Representing a Single Photon at time $t$

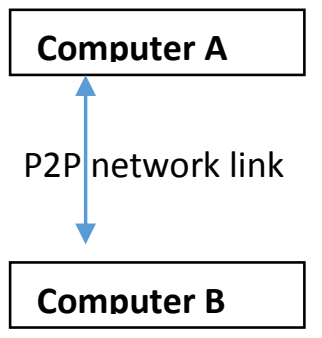

\section{Represented Photon Location}

Location A

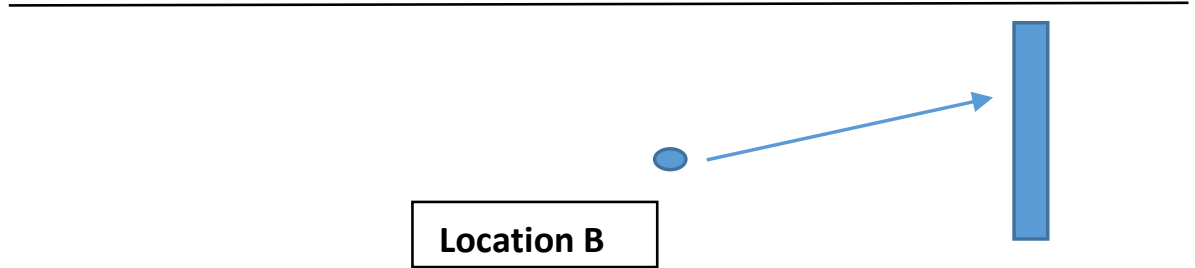

Half-silvered mirror

Each computer, in other words, represents the same particle as approaching the half-silvered mirror vis-à-vis a slightly different trajectory. Suppose next, then, that both computers process reality according to the following laws of nature:

If a particle approaches a half-silvered mirror from Location A, it passes through.

If a particle approaches a half-silvered mirror from Location B, it reflects backwards. Here, then, is what each computer will compute at time $t+1$ :

Figure 8. Two P2P-Linked Computers Representing a Single Photon at time $t+1$

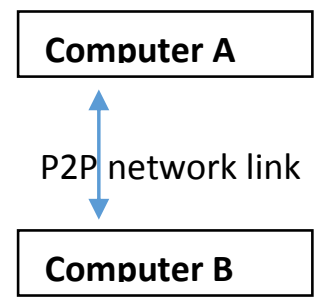
Represented Photon Location

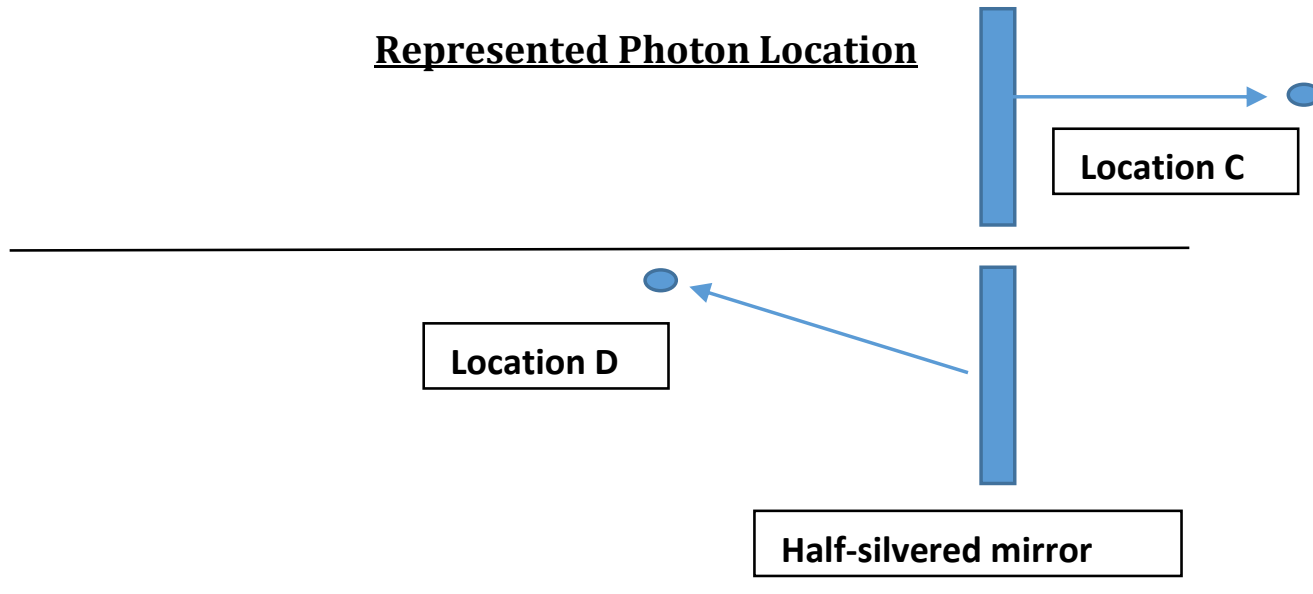


Each computer will-following the laws of nature it has encoded in its programmingrepresent "the photon" both had represented as approaching the half-silvered mirror at time t as now existing in very different positions after hitting the mirror: Computer A represents the photon as having passed through the barrier, and Computer B represents it as reflecting backward. Here is the next crucial step: both computers on the network represent that photon as a single entity from time $t$ to time $t+1$. Computer $A$, that is, represents "the photon" in their shared environment as now occupying Location $C$ on the other side of the mirror, whereas Computer B locates "the photon" in their environment as having reflected backwards to Location. And here is the final step: both computers are linked together in the P2P, so as to represent a shared environment. Computer A will tell computer B that "the photon" is at Location C on the other side of the mirror, and Computer B will tell computer A that, no, "the photon" has reflected backwards to Location D. Provided they have no further "tie-breaking" coding to determine which computer's location "wins", the end result will be that both computers will now tell each other to represent what was a single photon at time t now exists in two different places at time $t+1-$ as in:

Figure 9. Two P2P-Linked Computers Representing a Single Photon Post-informationtransfer "cross-talk" at t+1

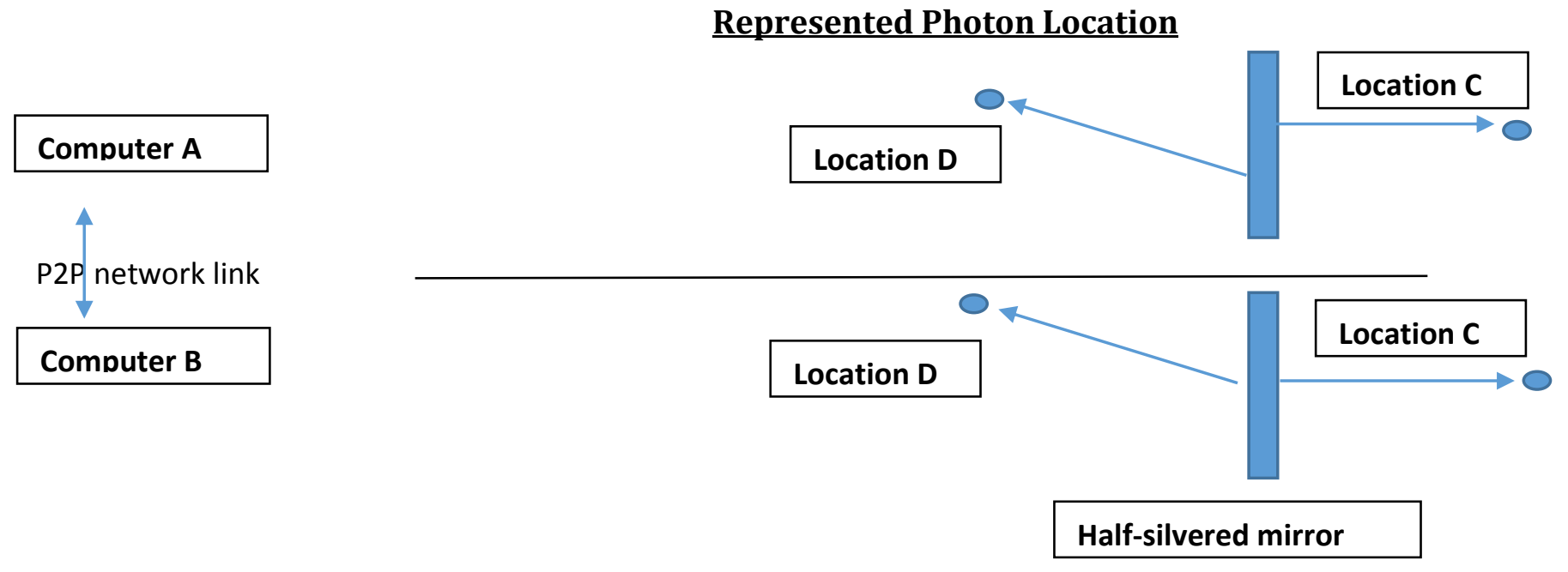


In other words, whereas both computers on the P2P network represented a single electron approaching the half-silvered mirror at $t,(\mathrm{~A})$ the differences in the approach vectors between them, coupled with (B) the informational cross-talk between the two computers (one represents the particle passing through the mirror and the other as bouncing back), results in (C) both computers representing what was previously a single photon as now (at $t+1$ ) a single photon in two places at once, where any intrinsic change to "one" just is a change to the "other" (since both computers represent them as being identical to the single photon that had approached the mirror). But this is precisely what quantum entanglement is in our world. Thus, the P2P Hypothesis explains quantum entanglement.

\section{§3. A New Research Program}

This paper has only sketched how the P2P Hypothesis promises to provide a unified explanation of features of our world that, until now, have left physicists and philosophers baffled. Quantum phenomena have long seemed "entirely counterintuitive." How, physicists and philosophers have asked, could reality be a superposition of states? What would the superposition be? Why would it be? Similarly, how could light, seemingly paradoxically, be a particle and a wave? What would explain its having both features simultaneously? Similarly, why does quantum indeterminacy exist? Why does the quantum wave-function appear to collapse upon the taking of a measurement? And why in the world are particles entangled across arbitrarily large distances?

All of these things have been a complete mystery-well, perhaps until now. The P2P Hypothesis may well turn out to be false. Yet I have just sketched how it appears to provide a single, intuitive explanation of all of the features of the quantum world that have left physicists and philosophers so baffled. If this is not a good reason for pursuing the 
hypothesis further-for using the methods of computer science, physics, mathematics, and philosophy-to construct P2P simulations, examine the manner(s) in which they might be programmed to give rise to specific features of quantum mechanics (e.g. the Standard Model of particle physics, etc.), I do not know what is. The P2P Hypothesis promises a deep, unified explanation of quantum phenomena. It should now fall to mathematicians, computer scientists, and physicists to formulate and investigate it at a more detailed level. Such investigations-by investigating the mathematics and computational structure of P2P Simulations-may well finally teach us why our reality is the way it is.

\section{Conclusion}

Our world displays what appear to be a bewildering array of strange quantum effects: quantum superposition, a wave-function "collapse" of such superpositions to determinate values upon measurement, fundamental indeterminacy, wave-particle duality, and entanglement. Existing physical and metaphysical theories explain none of these things, leaving them essentially as one grand mystery. The P2P Hypothesis appears to provide a unified explanation of them all. It is therefore a hypothesis worth taking seriously, and one to be investigated in an interdisciplinary manner, using all of the empirical and conceptual tools—philosophy, computer science, physics, and mathematics—at our disposal. 


\section{References}

Arvan, Marcus (2013). “A New Theory of Free Will”, The Philosophical Forum, 44(1): 1-49.

Brooks, Michael (2013). “Reality Checked.” New Scientist, 3 Aug. 2013: 33-36.

Cushing, James T. (1994). Quantum Mechanics: Historical Contingency and the Copenhagen Hegemony (University of Chicago Press).

Gates, James (2010). "Symbols of Power: Adinkras and the Nature of Reality”, Physics World, reprinted online at http://www.onbeing.org/program/uncovering-codesreality/feature/symbols-power-adinkras-and-nature-reality/1460 (accessed on 14 May 2013).

Schlosshauer, M., Kofler, J. and Zeilinger, A. (2013), The interpretation of quantum mechanics: from disagreement to consensus?. Ann. Phys., 525: A51-A54. doi: 10.1002/andp.201300722. 